\title{
Novel nomograms to predict lymph node metastasis and distant metastasis in resected patients with early-stage non-small cell lung cancer
}

\author{
Yi Tian ${ }^{1,2}, \mathrm{Yu} \mathrm{He}{ }^{2}, \mathrm{Xin}_{\mathrm{Li}^{3}}$, Xiaowen $\mathrm{Liu}^{1 \wedge}$ \\ ${ }^{1}$ Department of Oncology, Xiangya Hospital, Central South University, Changsha, China; ${ }^{2}$ Department of Thoracic Surgery, Second Xiangya \\ Hospital, Central South University, Changsha, China; ${ }^{3}$ Department of Dermatology, Second Xiangya Hospital, Central South University, Changsha, \\ China \\ Contributions: (I) Conception and design: X Liu, Y Tian; (II) Administrative support: X Liu; (III) Provision of study materials or patients: Y Tian, Y \\ He; (IV) Collection and assembly of data: Y Tian, Y He, X Li; (V) Data analysis and interpretation: Y Tian; (VI) Manuscript writing: All authors; (VII) \\ Final approval of manuscript: All authors. \\ Correspondence to: Xiaowen Liu. Department of Oncology, Xiangya Hospital, Central South University, No. 87 Xiangya Road, Changsha 410008 , \\ China. Email: xiaowenlable@gmail.com.
}

Background: We aimed to formulate and validate nomograms to unravel the significant risk factors
associated with lymph node metastasis (LNM) and distant metastasis in early-stage non-small cell lung
cancer (NSCLC).
Methods: Totally, 22403 pathologic T1 (pT1) or pT2 NSCLC patients were extracted from the
Surveillance, Epidemiology, and End Results (SEER) database and were further randomly assorted into
training and testing cohorts. Clinicopathologic risk predictors associated with distant metastasis and LNM
were investigated through multivariate logistic regression. The LNM and distant metastasis nomogram
models integrating all significant variables were internally validated in the testing cohort. The prediction
efficiency of nomograms was assessed via the receiver operating characteristics curve (ROC), calibration
plots and decision curve analysis (DCA). Results: The LNM nomogram displayed a favorable calibration and discrimination in both training and testing cohorts, with an area under the curve (AUC) of 0.721 (95\% CI: 0.711-0.734) and 0.713 (95\% CI: 0.699-0.727), respectively. And the encouraging prediction efficiency was also revealed in the training cohort (AUC $=0.788,95 \%$ CI: 0.761-0.816) and the testing cohort (AUC $=0.765,95 \%$ CI: $0.721-0.808$ ) for the distant metastasis nomogram. The calibration plot revealed an optimal consistency between model prediction and practical observation. Both nomograms were endowed with the optimal clinical utility and benefits compared with conventional clinicopathological indicators.

Conclusions: Our nomogram models are a promising tool with robust predictive power to effectively and intuitively predict the occurrence of LNM and distant metastasis in early-stage NSCLC.

Keywords: SEER; early-stage non-small cell lung cancer (NSCLC); lymph node metastasis (LNM); nomogram

Submitted Sep 01, 2020. Accepted for publication Nov 23, 2020.

doi: 10.21037/apm-20-1756

View this article at: http://dx.doi.org/10.21037/apm-20-1756

^ORCID: 0000-0002-2543-4599. 


\section{Introduction}

Lung cancer is the most common cause of cancer-related death globally (1). About 1.8 million patients are estimated to die from lung cancer in 2018 (2). Non-small cell lung cancer (NSCLC) is the most dominating histologic subtype of all primary lung cancers, occupying about $85 \%$ of all cases $(3,4)$. NSCLC is pathologically categorized into squamous cell carcinoma, adenocarcinoma, and large cell carcinoma (5). The 5-year survival rates of lung cancer fluctuate ranging from $4 \%$ to $17 \%$, which is primarily ascribed to tumor stage and regional discrepancy $(6,7)$. The unsatisfactory prognosis and frequent recurrence of NSCLC are closely associated with lymph node metastasis (LNM) and distant metastasis (8).

In accordance with the 7th edition of the TNM staging system of lung cancer $(9,10)$, patients can be diagnosed with advanced NSCLC (TNM stage IV) in the presence of distant metastasis, regardless of the pathologic $\mathrm{T}$ (pT) classification (10). NSCLC is characterized with formidable metastatic capacity and the bone (25-30\%) is the most frequent metastatic site, followed by the lung (20-25\%), the brain (15-20\%) and the liver (5-10\%) $(11,12)$. Approximately $70-80 \%$ of patients diagnosed with NSCLC develop LNM in the clinical course of their disease. Furthermore, approximately $40-50 \%$ of advanced NSCLC patients (TNM stage III and IV) remain at pT1 or pT2 because of the aggressive characteristics of earlystage NSCLC $(13,14)$. Above mentioned patients merely have 5 -year survival rates of $4-15 \%$ on account of the progression of distant metastasis and subsequent palliative systemic treatment $(6,14)$. Specifically, NSCLC patients with liver metastasis and abdominal lymph nodes metastasis are correlated with significantly diminished overall survival (OS) (15). For NSCLC patients with a pathological tumor size less than $30 \mathrm{~mm}$, cases with LNM tend to display a much more unsatisfactory median OS than those without LNM (54.4 versus 114.1 months, $\mathrm{P}<0.001$ ) (16). Thus, distant metastasis and LNM are crucial determinants of the prognosis of early-stage NSCLC (pT1 or pT2). Early detection of metastasis in early-stage NSCLC is essential for improving therapeutic planning and patient's risk stratification in clinical practice.

Currently, there is merely confined information concerning how to estimate the risk factors related to LNM and distant metastasis in early-stage NSCLC individuals. Therefore, we attempted to develop and identify graphical calculating nomograms with an integration of all significant clinicopathological variables, thus accurately quantifying the risk events for tumor progression $(17,18)$. To date, nomograms have not been formulated to predict LNM and distant metastasis in patients with early-stage NSCLC through extracting clinical data from the Surveillance, Epidemiology, and End Results (SEER) database. Herein, our study was designed to establish the first nomograms to confer risk prediction of LNM and distant metastasis in resected early-stage NSCLC patients.

We present the following article in accordance with the TRIPOD reporting checklist (available at http://dx.doi. org/10.21037/apm-20-1756).

\section{Methods}

\section{Data source and population selection}

The SEER program financed by the National Cancer Institute is composed of 18 population-based cancer registries, covering approximately $28 \%$ of the U.S. population $(19,20)$. The study was conducted in accordance with the Declaration of Helsinki (as revised in 2013). Considering that all available information from the public SEER database was anonymized, our study was exempted from the ethics committee approval and consent procedure $(20,21)$. Because the SEER database initiated collecting the information for metastatic sites of bone, liver, lung and brain in 2010 and relevant data was renewed until 2015, we queried all pT1 or pT2a NSCLC patients who underwent resection between 2010 and 2015 from the SEER database through SEER ${ }^{*}$ Stat 8.3.6 software. The flowchart of patient selection was shown in Figure 1.

A total of 20 clinical parameters were extracted for each patient, including demographic variables (age at diagnosis, sex, ethnicity and survival time), tumorassociated characteristics (primary tumor sites, pathological differentiation, pT stage, $\mathrm{N}$ stage, $\mathrm{M}$ stage, TNM stage, tumor size, histological type, pleural invasion, the status of distant metastasis, lung metastasis, bone metastasis, brain metastasis, liver metastasis) and cancer-targeted therapy (surgery type). Specifically, histological classifications of NSCLC, including "Adenocarcinoma", "Squamous cell carcinoma", "Large cell lung cancer (LCLC)" and "NSCLC, Not otherwise specified (NSCLC/NOS)" were confirmed based on the International Classification of Diseases for Oncology, 3rd Edition (IDO-O-3). All NSCLC cases were staged based on the 7th edition of the TNM staging system. The exclusion criteria were as follows: patients who 




Figure 1 Flowchart of patient selection. NSCLC, non-small cell lung cancer; pT, pathologic T.

aged $<18$ years old or were diagnosed merely by autopsy or death certificate, or whose clinical data were insufficient. Eventually, a total of 22,403 enrolled cases extracted from the SEER database were randomly classified into a training cohort $(n=14,935)$ for nomogram establishment and a testing cohort $(\mathrm{n}=7,468)$ for internal verification.

\section{Establishment and validation of nomogram models}

All significant independent risk factors for LNM and distant metastasis were confirmed in early-stage NSCLC cases through univariable and multivariable logistic regression analysis $(\mathrm{P}<0.05)$. The SEER internal testing cohort was applied for evaluating the predictive performance of the nomograms associated with LNM and distant metastasis. A bootstrapping strategy with 2,000 samples was further conducted for the internal validation of the nomogram models. The receiver operating characteristics curve (ROC) and calibration plots were used to measure and validate the predictive reliability and accuracy of the models, respectively. Subsequently, decision curve analysis (DCA) curve was established to evaluate the clinical utility and benefits of the nomogram and other conventional clinicopathological parameters.

\section{Statistical analysis}

The $\mathrm{R}$ language version 3.3.5 was applied for statistical analyses. Means and standard deviation were utilized to represent continuous variables. Variables could be compared through the Chi squared test and Student's $t$-test. The nomogram models and the corresponding calibration plots as well as ROC curve were calculated by the pROC and rms packages. The cutoff values of the risk scores in the predictive nomogram models were estimated through the maximum Youden index. All cases were further classified into low- and high-risk groups. All tests were 2-tailed and considered statistically significant at $\mathrm{P}<0.05$.

\section{Results}

\section{Clinicopathologic features of patients}

As shown in Table S1, there were no statistically significant discrepancies in the demographic and clinical characteristics of early-stage NSCLC patients between the training and the validation cohorts $(\mathrm{P}>0.05)$. LNM occurred in 1,653 of 14,935 patients $(11.06 \%)$ in the training group and 697 of 7,468 patients $(9.33 \%)$ in testing group. Distant metastasis was present in 261 of 14,935 patients $(1.75 \%)$ in the training cohort and 131 of 7,468 patients $(1.75 \%)$ in the testing cohort. Specifically, in the training cohort, the proportion of bone metastasis, brain metastasis, liver metastasis was $0.42 \%(\mathrm{n}=63), 0.91 \%(\mathrm{n}=136), 0.15 \%(\mathrm{n}=22)$, respectively. The corresponding numerical value in the testing cohort was $0.43 \%(n=32), 0.99 \%(n=74), 0.09 \%(n=7)$, respectively. Table S1 revealed no statistically significant discrepancy in 
LNM proportion $(\mathrm{P}=0.47)$ or distant metastasis proportion $(\mathrm{P}=1.00)$ between both groups.

\section{Correlations between clinicopathologic parameters and LNM and distant metastasis}

The existence of LNM was examined stratified by different clinicopathologic variables. Among all clinical parameters, LNM was significantly correlated with age at diagnosis, primary site, pathological differentiation, pT stage, metastasis status, tumor size, pleural invasion, surgery type, brain metastasis, bone metastasis and lung metastasis as well as OS in both the training and testing cohorts $(\mathrm{P}<0.001)$ (Table 1). Similarly, in the correlation analysis, 11 parameters, namely age, pathological differentiation, lymph node status, histological type, pleural invasion, surgery type, brain metastasis, bone metastasis, liver metastasis and lung metastasis as well as OS were all significantly associated with distant metastasis in two sets $(\mathrm{P}<0.001)$ (Table 2).

\section{Univariable and multivariable logistic regression analysis in training cohort}

Initially, we preliminarily screened the independent risk factors for LNM and distant metastasis through univariate logistic regression analysis (Table 3). LNM was significantly correlated with age, gender, ethnicity, primary site, pathological differentiation, $\mathrm{pT}$ stage, tumor size, histological type, pleural invasion, surgery type and distant metastasis $(\mathrm{P}<0.05)$. Additionally, age, gender, pathological differentiation, pT stage, lymph node status, histological type, pleural invasion and surgery type were significantly related to distant metastasis of early-stage NSCLC $(\mathrm{P}<0.05)$.

The abovementioned significant risk predictors in the univariate analysis were all incorporated into the multivariate analysis. The associated risk factors of LNM and distant metastasis and the corresponding OR with 95\% CI were presented in Table 4. Over 65 years of age $(\mathrm{OR}=0.77,95 \% \mathrm{CI}: 0.67-0.81, \mathrm{P}<0.001)$ was negatively associated with the development of LNM. Furthermore, for histological type, patients with NSCLC-subtype squamous cell carcinoma had a significantly diminished risk of LNM compared with those with adenocarcinoma $(\mathrm{OR}=0.61,95 \%$ CI: 0.55-0.68, $\mathrm{P}<0.001)$. Nevertheless, male gender (OR $=1.12,95 \%$ CI: $1.02-1.22, \mathrm{P}=0.015)$, T2a stage $(\mathrm{OR}=1.59$, 95\% CI: 1.36-1.86, $\mathrm{P}<0.001)$, tumor size of $30-50 \mathrm{~mm}$ (OR $=1.47,95 \%$ CI:1.28-1.69, $\mathrm{P}<0.001)$ and distant metastasis $(\mathrm{OR}=2.94,95 \% \mathrm{CI}: 2.25-3.83, \mathrm{P}<0.001)$ were positively associated with LNM. As for primary site of early-stage NSCLC, compared with right upper lobe (RUL), tumor located in the left lower lobe (LLL) displayed the highest risk of $\mathrm{LNM}(\mathrm{OR}=1.37,95 \% \mathrm{CI}: 1.2-1.57, \mathrm{P}<0.001)$, followed by left upper lobe (LUL) $(\mathrm{OR}=1.36,95 \% \mathrm{CI}$ : $1.22-1.52, \mathrm{P}<0.001)$, right middle lobe $(\mathrm{RML})(\mathrm{OR}=1.33$, 95\% CI: $1.1-1.59, \mathrm{P}=0.003$ ) and right lower lobe (RLL) (OR $=1.31,95 \%$ CI: $1.16-1.49, \mathrm{P}<0.001)$. NSCLC patients with moderately or poorly-differentiated tumors were more prone to develop LNM than those with well-differentiated tumors $(\mathrm{OR}=2.96,95 \% \mathrm{CI}: 2.54-3.46, \mathrm{P}<0.001$ for moderately-differentiated tumors; and $\mathrm{OR}=4.19,95 \% \mathrm{CI}$ : 3.59-4.91, $\mathrm{P}<0.001$ for poorly-differentiated tumors).

Multivariable logistic regression indicated that age $>65$ years old $(\mathrm{OR}=0.53,95 \% \mathrm{CI}: 0.41-0.68, \mathrm{P}=0.006)$ and squamous cell carcinoma ( $\mathrm{OR}=0.44,95 \% \mathrm{CI}: 0.3-0.63$, $\mathrm{P}<0.001)$ were negatively correlated with the occurrence of distant metastasis (Table 4). Early-stage NSCLC patients treated with lobectomy/bilobectomy $(\mathrm{OR}=0.35,95 \% \mathrm{CI}$ : $0.26-0.46, \mathrm{P}<0.001)$ or pneumonectomy $(\mathrm{OR}=0.33,95 \%$ CI: $0.13-0.7, \mathrm{P}<0.001)$ showed a significantly diminished risk of distant metastasis compared to those treated with wedge resection. Conversely, male patients $(\mathrm{OR}=1.43$, 95\% CI: $1.11-1.85, \mathrm{P}<0.001)$ with pleural invasion $(\mathrm{OR}=1.66$, 95\% CI: $1.15-2.42, \mathrm{P}<0.001)$ exhibited an increased risk for developing distant metastasis. Moderately $(\mathrm{OR}=2.97$, 95\% CI: 1.74-5.43, $\mathrm{P}<0.001)$ or poorly-differentiated (OR $=4.96,95 \%$ CI: $2.92-9.05, \mathrm{P}<0.001)$ tumors had a higher risk of the development of distant metastasis than welldifferentiated tumors. For lymph node status, patients with more aggressive lymph node involvement had a higher risk of distant metastasis $(\mathrm{OR}=1.98,95 \%$ CI: $1.33-2.88$, $\mathrm{P}<0.001$ for $\mathrm{N} 1$; OR $=3.11$, 95\% CI: $2.26-4.23, \mathrm{P}<0.001$ for $\mathrm{N} 2$; OR $=15.14,95 \%$ CI: 7.01-31.4, $\mathrm{P}<0.001$ for N3).

\section{Development of nomogram model for LNM and distant metastasis prediction}

Significant results of the multivariate regression model were applied for constructing nomogram models of LNM (Figure 2) and distant metastasis (Figure 3). Moreover, Table S2 revealed the estimated points scales and predictive scores for each parameter in the nomograms. Specifically, Figure 2 demonstrated that surgery type and degrees of pathological differentiation made the greatest contribution to the presence of LNM, followed by the status of distant metastasis, pT stage, and histological type as well as tumor size. The primary site of tumor, age and gender exerted a 
Table 1 Correlations between clinicopathological variables and LNM in both cohorts



Table 1 (continued) 
Table 1 (continued)

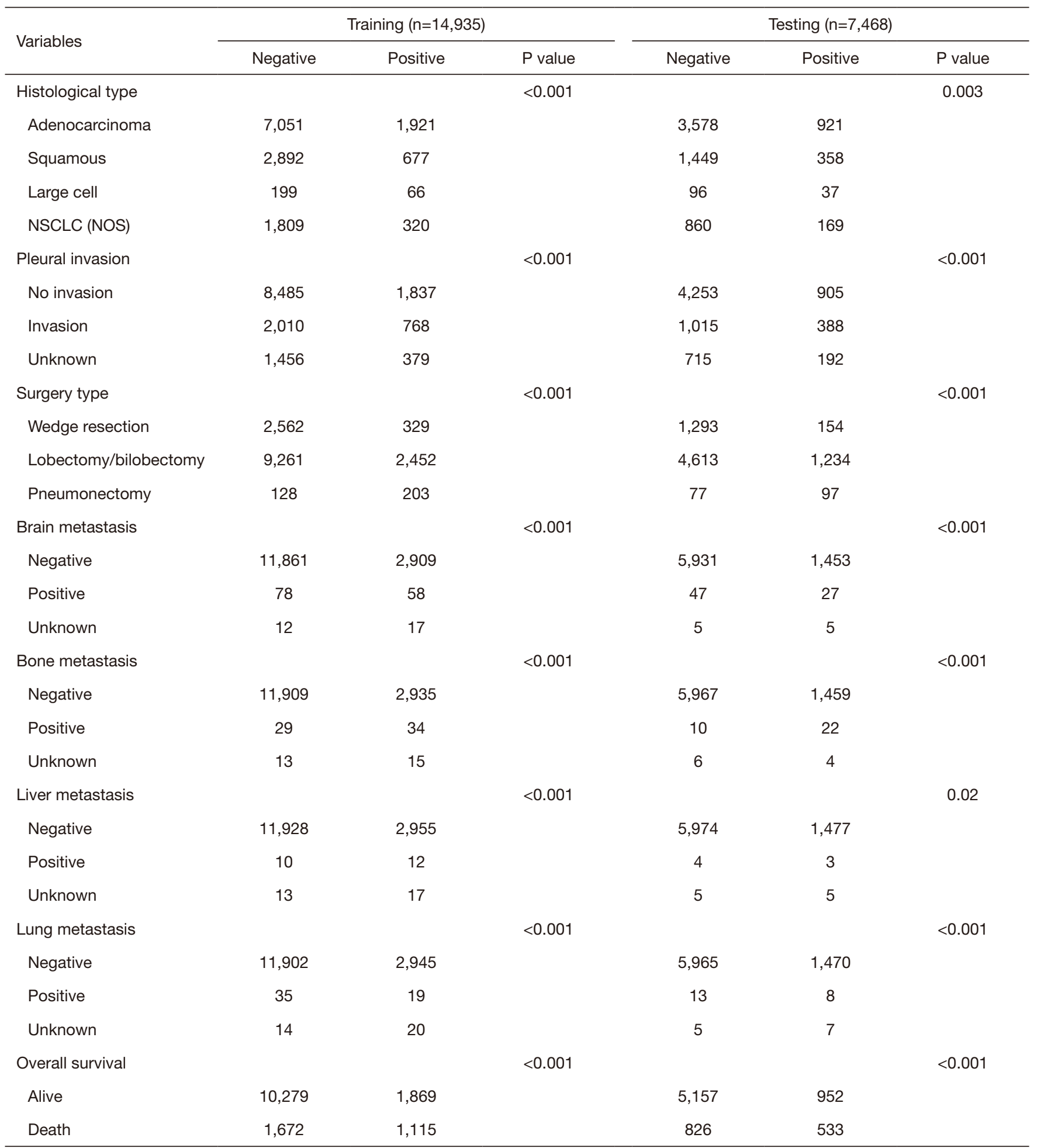

LNM, lymph node metastasis; SD, standard deviation; pT, pathologic T; RUL, right upper lobe; RML, right middle lobe; RLL, right lower lobe; LUL, left upper lobe; LLL, left lower lobe; pT, pathologic T; NSCLC (NOS), non-small cell lung cancer, not otherwise specified. 
Table 2 Correlations between clinicopathological variables and distant metastasis in both cohorts



Table 2 (continued) 
Table 2 (continued)

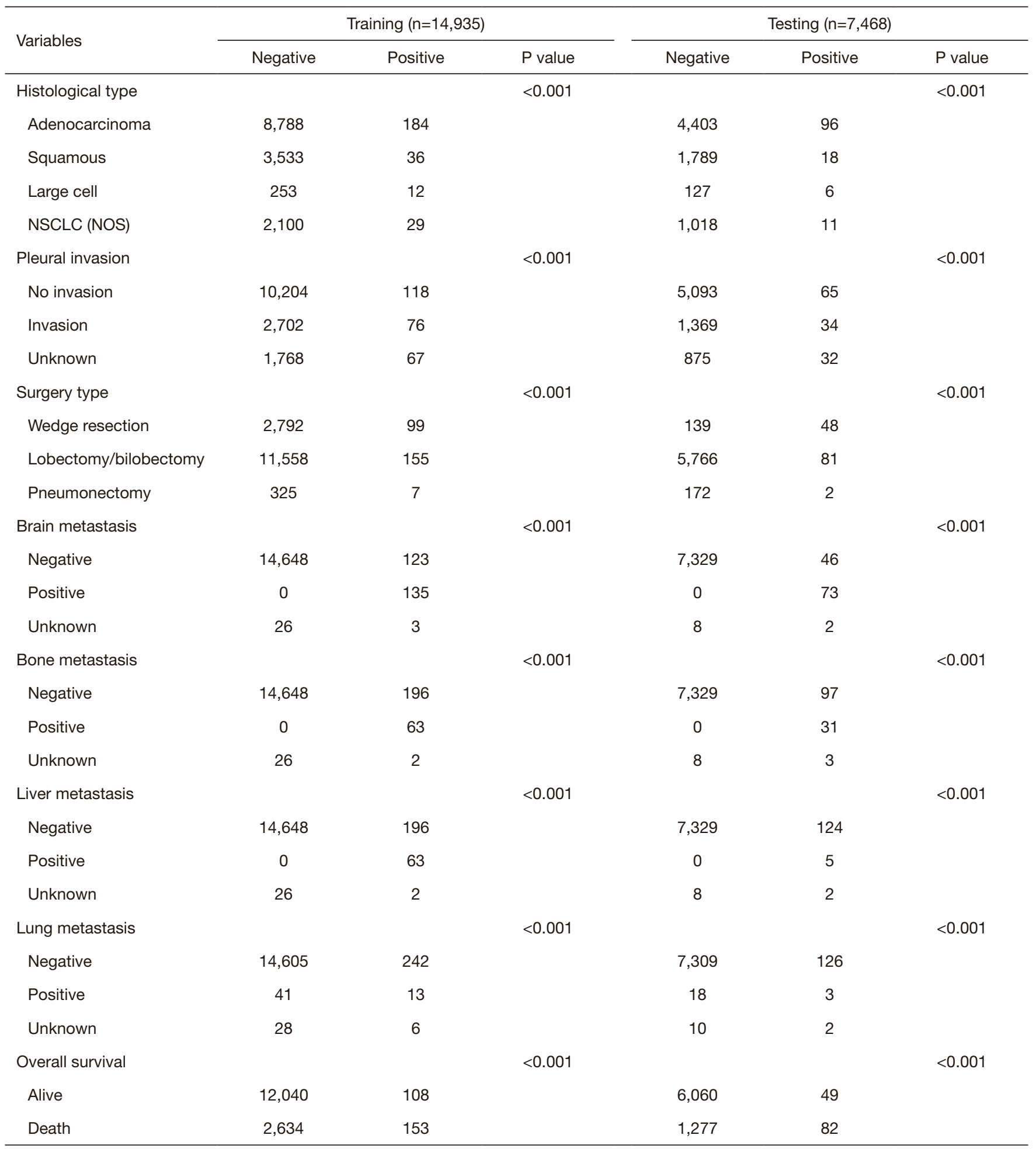

*, NX indicates unknown stage. RUL, right upper lobe; RML, right middle lobe; RLL, right lower lobe; LUL, left upper lobe; LLL, left lower lobe; pT, pathologic T; NSCLC (NOS), non-small cell lung cancer, not otherwise specified. 
Table 3 Univariate logistic regression to analyze risk factors for LNM and distant metastasis

\begin{tabular}{|c|c|c|c|c|c|c|}
\hline Variables & \multicolumn{3}{|c|}{ LNM } & \multicolumn{3}{|c|}{ Distant metastasis } \\
\hline \multicolumn{7}{|l|}{ Age } \\
\hline$\leq 65$ & 1 & & & 1 & & \\
\hline$>65$ & 0.71 & $0.65-0.77$ & $<0.001$ & 0.52 & $0.4-0.66$ & $<0.001$ \\
\hline Female & 1 & & & 1 & & \\
\hline Male & 1.28 & $1.17-1.38$ & $<0.001$ & 1.53 & $1.2-1.97$ & $<0.001$ \\
\hline \multicolumn{7}{|l|}{ Race } \\
\hline White & 1 & & & 1 & & \\
\hline RUL & 1 & & & 1 & & \\
\hline RML & 1.19 & $1-1.42$ & 0.05 & 1.08 & $0.62-1.78$ & 0.77 \\
\hline RLL & 1.18 & $1.04-1.33$ & 0.007 & 0.76 & $0.5-1.12$ & 0.18 \\
\hline LUL & 1.36 & $1.22-1.51$ & $<0.001$ & 1.29 & $0.95-1.75$ & 0.11 \\
\hline LLL & 1.28 & $1.13-1.46$ & $<0.001$ & 0.94 & $0.63-1.39$ & 0.77 \\
\hline \multicolumn{7}{|c|}{ Pathological differentiation } \\
\hline Well; I & 1 & & & 1 & & \\
\hline Moderately; II & 3.15 & $2.73-3.64$ & $<0.001$ & 3.23 & $1.94-5.78$ & $<0.001$ \\
\hline \multicolumn{7}{|l|}{ Lymph node status } \\
\hline No & - & - & - & 1 & & \\
\hline $\mathrm{N} 1$ & - & - & - & 2.08 & $1.42-2.96$ & $<0.001$ \\
\hline $\mathrm{N} 2$ & - & - & - & 4.28 & $3.16-5.74$ & $<0.001$ \\
\hline N3 & - & - & - & 42.18 & $21.2-80.5$ & $<0.001$ \\
\hline \multicolumn{7}{|l|}{ Tumor size } \\
\hline$\leq 30 \mathrm{~mm}$ & 1 & & & 1 & & \\
\hline $30-50 \mathrm{~mm}$ & 2.54 & $2.34-2.77$ & $<0.001$ & 1.31 & $1-1.69$ & 0.05 \\
\hline
\end{tabular}

Table 3 (continued) 
Table 3 (continued)

\begin{tabular}{|c|c|c|c|c|c|c|}
\hline Variables & \multicolumn{3}{|c|}{ LNM } & \multicolumn{3}{|c|}{ Distant metastasis } \\
\hline \multicolumn{7}{|l|}{ Histological type } \\
\hline Adenocarcinoma & 1 & & & 1 & & \\
\hline Squamous & 0.86 & $0.78-0.95$ & 0.002 & 0.49 & $0.33-0.69$ & $<0.001$ \\
\hline NSCLC (NOS) & 0.65 & $0.57-0.74$ & $<0.001$ & 0.66 & $0.44-0.96$ & 0.04 \\
\hline \multicolumn{7}{|l|}{ Pleural invasion } \\
\hline No invasion & 1 & & & 1 & & \\
\hline Invasion & 1.76 & $1.6-1.94$ & $<0.001$ & 2.43 & $1.81-3.25$ & $<0.001$ \\
\hline Lobectomy/bilobectomy & 2.06 & $1.83-2.34$ & $<0.001$ & 0.38 & $0.29-0.49$ & $<0.001$ \\
\hline Pneumonectomy & 12.35 & $9.64-15.9$ & $<0.001$ & 0.61 & $0.25-1.23$ & 0.21 \\
\hline \multicolumn{7}{|l|}{ Distant metastasis } \\
\hline Negative & 1 & & & - & - & - \\
\hline Positive & 3.56 & $2.78-4.56$ & $<0.001$ & - & - & - \\
\hline
\end{tabular}

LNM, lymph node metastasis; RUL, right upper lobe; RML, right middle lobe; RLL, right lower lobe; LUL, left upper lobe; LLL, left lower lobe; pT, pathologic T; NSCLC (NOS), non-small cell lung cancer, not otherwise specified.

Table 4 Multivariable logistic regression to analyze risk factors for LNM and distant metastasis

\begin{tabular}{|c|c|c|c|c|c|c|}
\hline Variables & \multicolumn{3}{|c|}{ LNM } & \multicolumn{3}{|c|}{ Distant metastasis } \\
\hline \multicolumn{7}{|l|}{ Age } \\
\hline$\leq 65$ & 1 & & & 1 & & \\
\hline$>65$ & 0.77 & $0.67-0.81$ & $<0.001$ & 0.53 & $0.41-0.68$ & 0.006 \\
\hline Female & 1 & & & 1 & & \\
\hline Male & 1.12 & $1.02-1.22$ & 0.015 & 1.43 & $1.11-1.85$ & $<0.001$ \\
\hline \multicolumn{7}{|c|}{ Primary site } \\
\hline RUL & 1 & & & - & - & - \\
\hline LUL & 1.36 & $1.22-1.52$ & $<0.001$ & - & - & - \\
\hline LLL & 1.37 & $1.2-1.57$ & $<0.001$ & - & - & - \\
\hline
\end{tabular}

Table 4 (continued) 
Table 4 (continued)

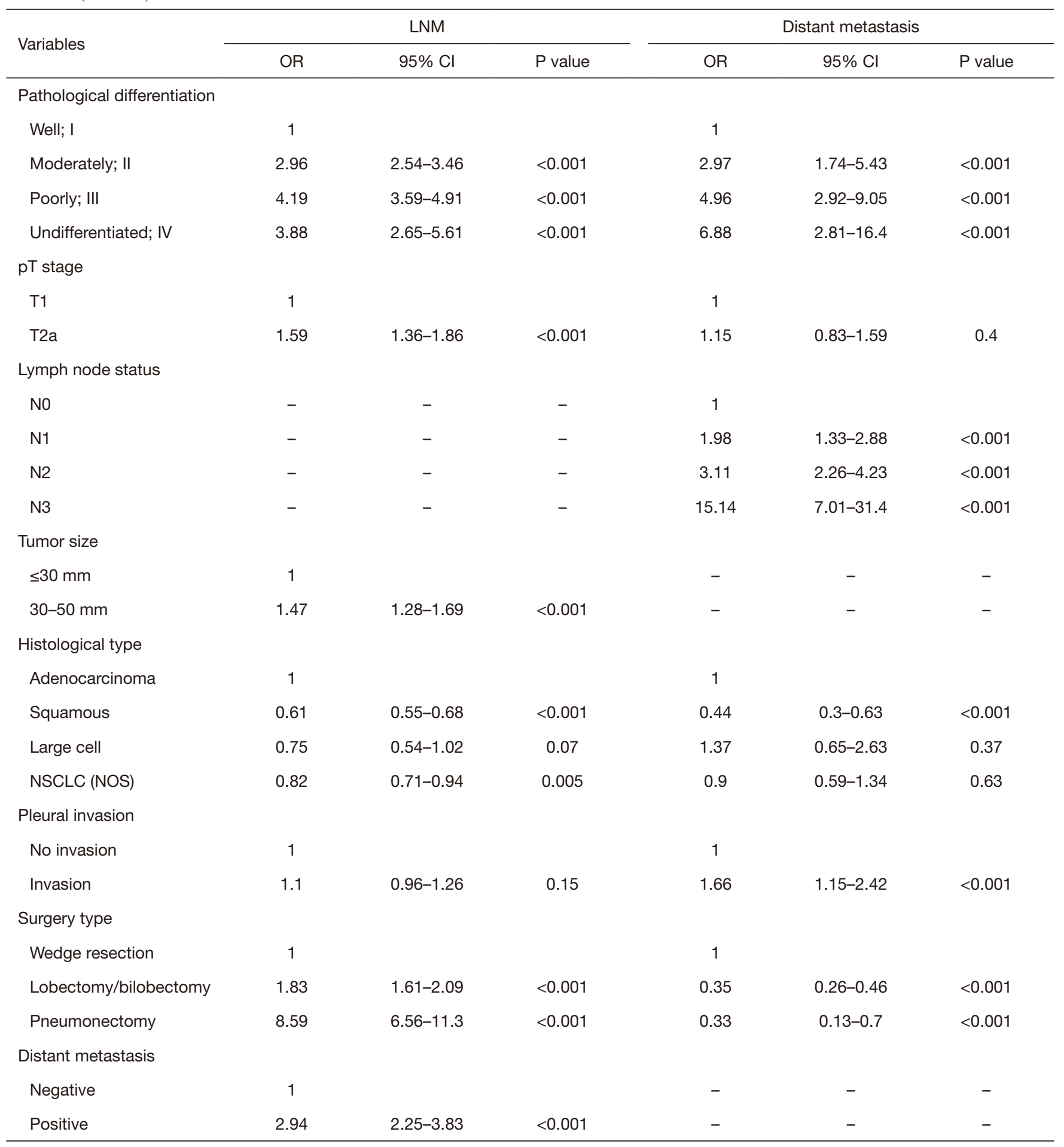

LNM, lymph node metastasis; RUL, right upper lobe; RML, right middle lobe; RLL, right lower lobe; LUL, left upper lobe; LLL, left lower lobe; pT, pathologic T; NSCLC (NOS), non-small cell lung cancer, not otherwise specified. 
A

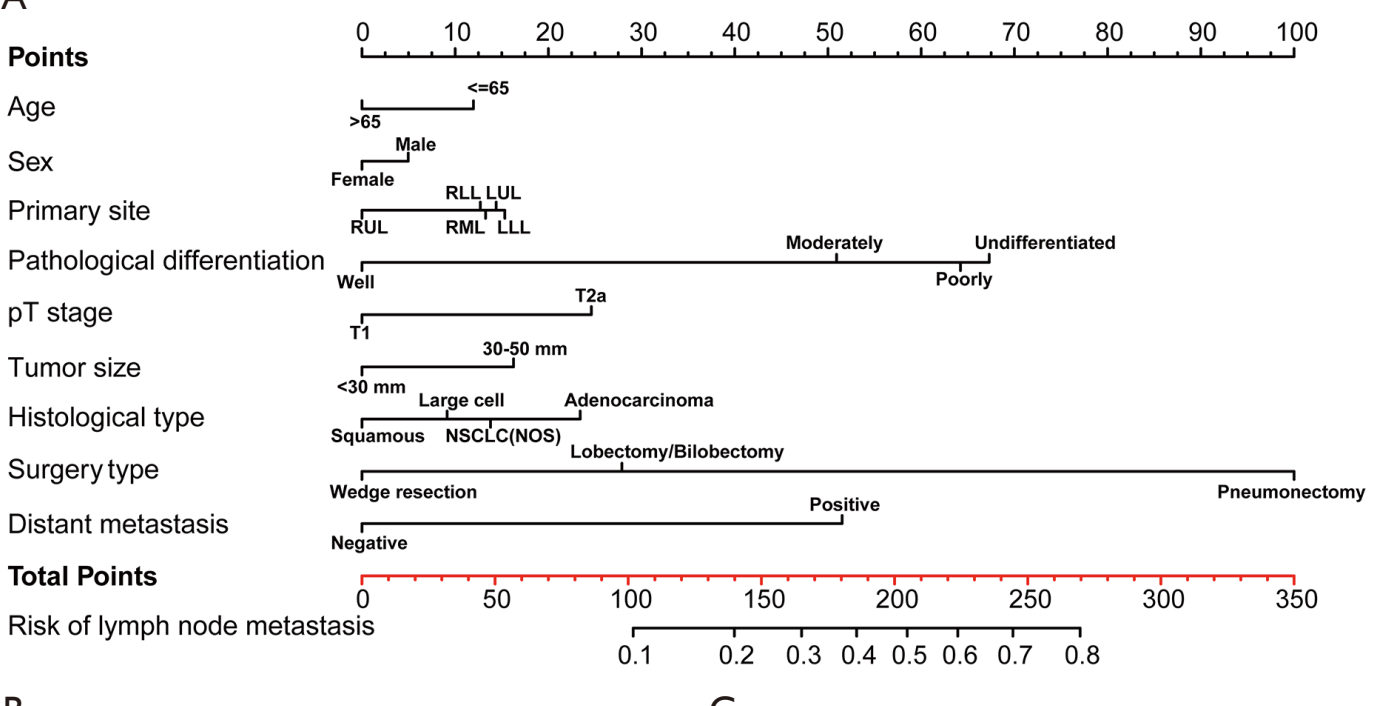

B

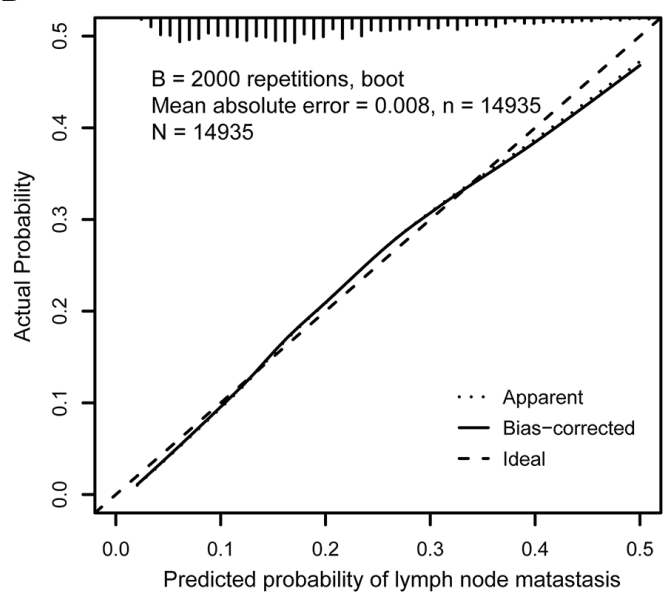

C

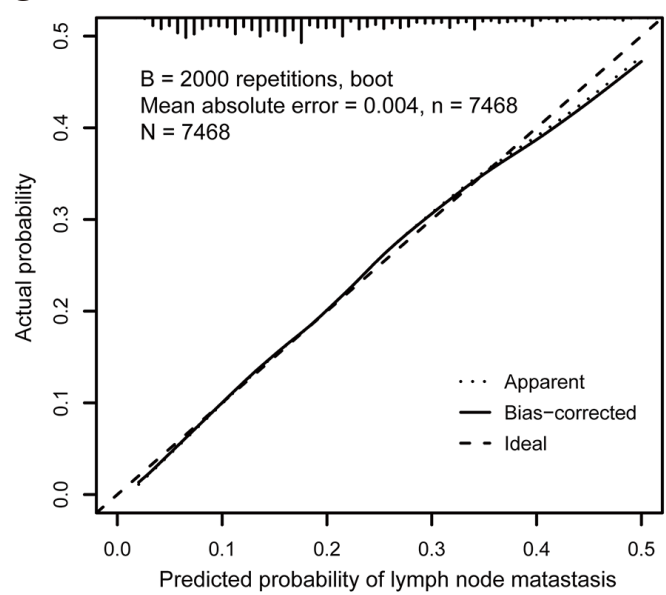

Figure 2 Nomogram models and calibration plots to predict LNM in early-stage NSCLC patients. (A) Nomograms for predicting the risk factors responsible for LNM. Calibration curves to estimate the predictive performance of LNM nomogram in (B) the training cohort and (C) the testing cohort, respectively. LNM, lymph node metastasis; RUL, right upper lobe; RML, right middle lobe; RLL, right lower lobe; LUL, left upper lobe; LLL, left lower lobe; pT, pathologic T; NSCLC (NOS), non-small cell lung cancer, not otherwise specified.

relatively small effect on the risk of LNM. Similarly, the status of lymph node invasion and degrees of pathological differentiation exerted the most noticeable impact on the distant metastasis nomogram, followed by histological type, surgery type, the status of pleural invasion. Age and sex made a few contributions to the risk of distant metastasis (Figure 3).

\section{Efficiency and validation of nomogram model for LNM and distant metastasis prediction}

The calibration plots to predict LNM (Figure 2) and distant metastasis (Figure 3) revealed a favorable concordance between the predicted and observed probabilities in the training and testing set, respectively. We performed a comparison of the predictive accuracy for LNM and distant metastasis between the nomograms and clinicopathological parameters through ROC observation and its corresponding area under the curve (AUC). The predictive value of the LNM nomogram was favorable, achieving an AUC of 0.721 (95\% CI: 0.711-0.734) in the training group (Figure 4) and 0.713 (95\% CI: 0.699-0.727) in the testing cohort (Figure 4), respectively, which were more satisfactory than the AUCs of pathological 
A

\section{Points}

Sex

Age

Pathological differentiation

Lymph node stage

Histological type

Pleural invasion

Surgery type

Total Points

Risk of distant metastasis

B
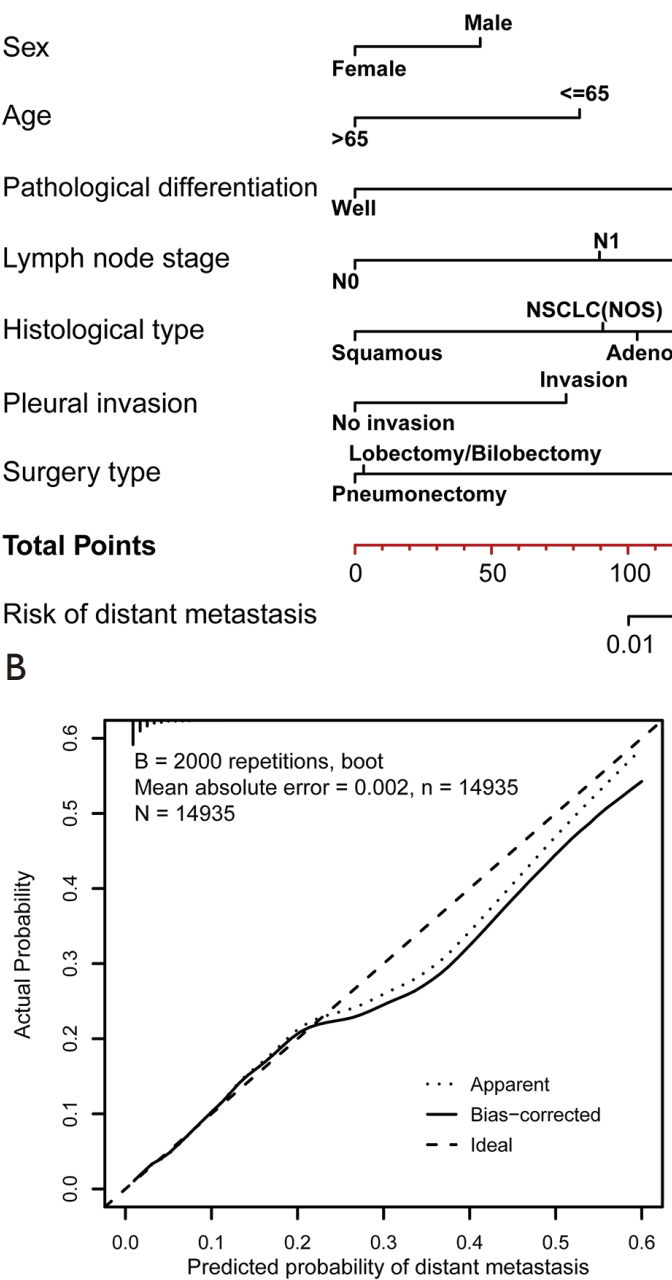

N1
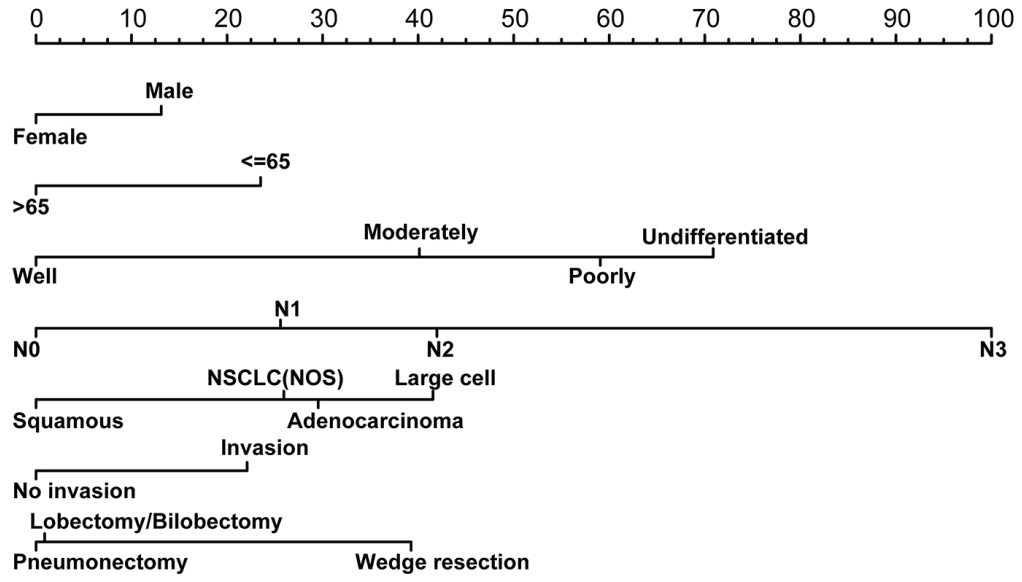

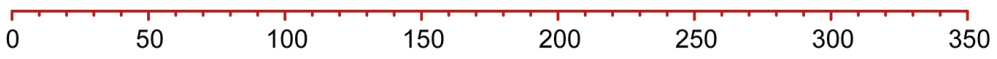

C

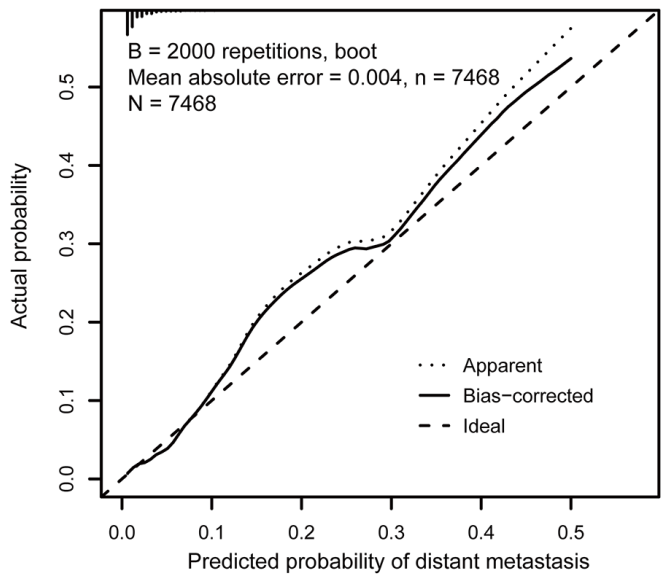

Figure 3 Nomogram models and calibration plots to predict distant metastasis in early-stage NSCLC patients. (A) Nomograms for predicting the risk factors responsible for distant metastasis. Calibration curves to estimate the predictive performance of distant metastasis nomogram in (B) the training cohort and (C) the testing cohort, respectively. NSCLC (NOS), non-small cell lung cancer, not otherwise specified.

differentiation, $\mathrm{pT}$ stage and histological type in two cohorts $(\mathrm{P}<0.001)$. Analogously, the distant metastasis nomogram model exhibited an AUC value of 0.788 (95\% CI: 0.761-0.816) in the training cohort (Figure 4) and 0.765 (95\% CI: 0.721-0.808) in the testing cohort (Figure 4), respectively. These values were higher than those for pathological differentiation, pleural invasion, the status of lymph node invasion and histological type. These results highlighted that the nomogram models conferred a more desirable predictive efficacy than that of the clinicopathological risk factors alone.

Additionally, the bar charts were formulated to assess the discriminatory efficiency of the LNM and distant metastasis nomogram models following estimating the risk scores. Based on the maximum Youden index, we further established optimal risk score cutoff values of 130 and 102 for the LNM and distant metastasis nomogram, respectively. All enrolled subjects were stratified into lowand high-risk groups. In the training cohort, early-stage NSCLC individuals in the high-risk group had a higher 
A

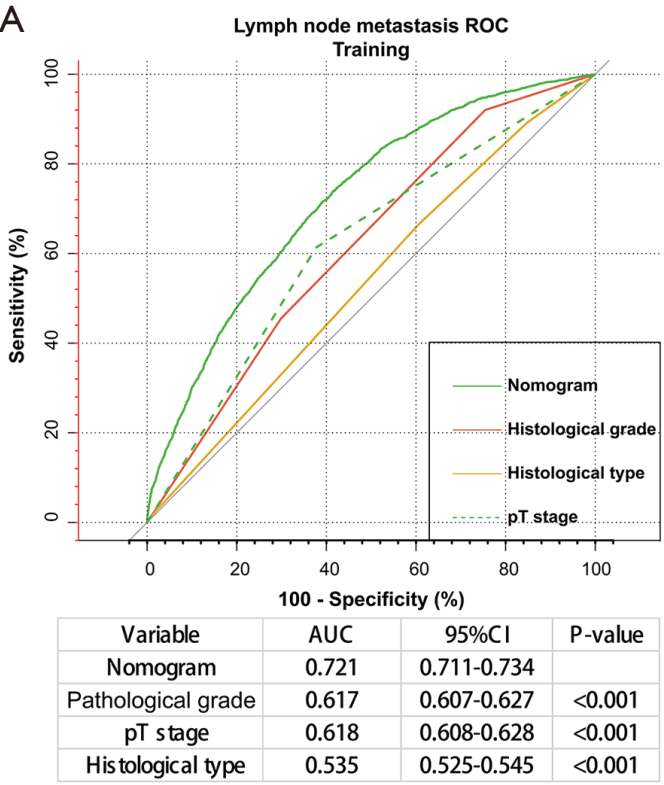

C

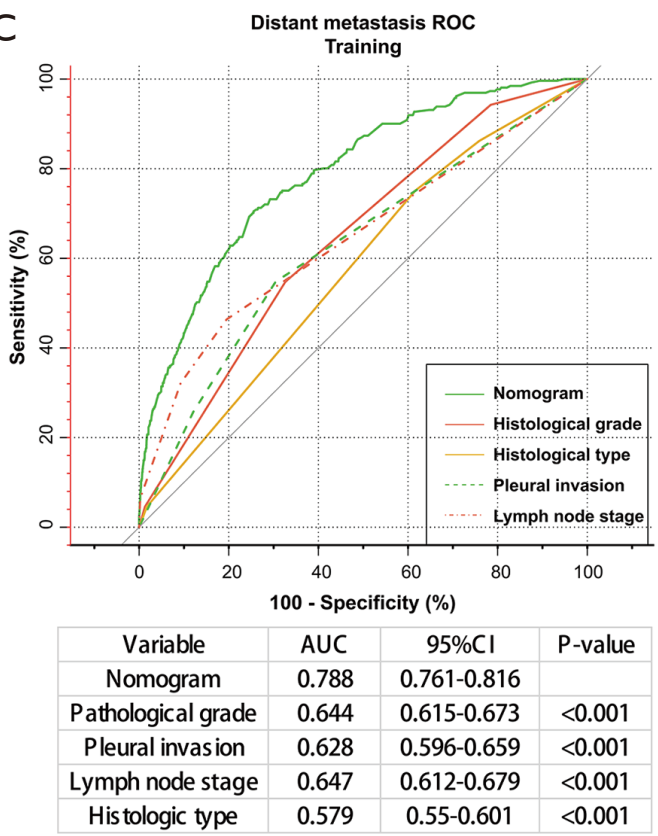

B


D

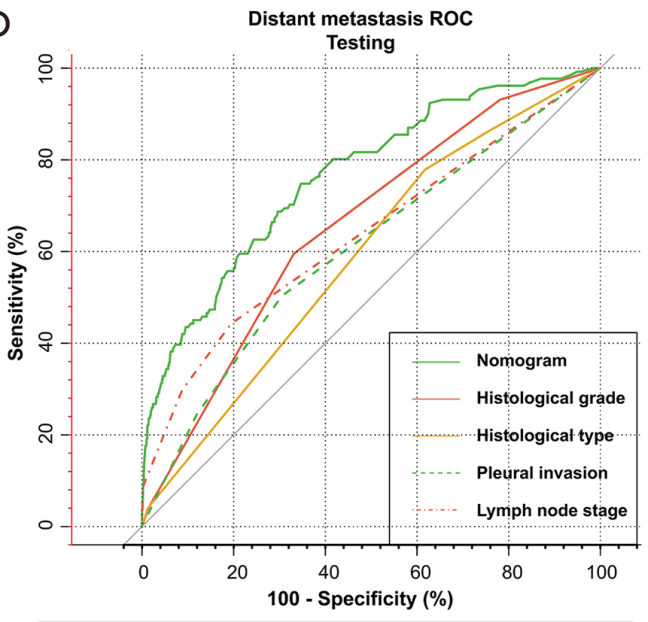

\begin{tabular}{|c|c|c|c|}
\hline Variable & AUC & $95 \% \mathrm{Cl}$ & $\mathrm{P}$-value \\
\hline Nomogram & 0.765 & $0.721-0.808$ & \\
\hline Pathological grade & 0.656 & $0.615-0.697$ & $<0.001$ \\
\hline Pleural invasion & 0.606 & $0.561-0.652$ & $<0.001$ \\
\hline Lymph node stage & 0.637 & $0.592-0.683$ & $<0.001$ \\
\hline His tologic type & 0.589 & $0.549-0.628$ & $<0.001$ \\
\hline
\end{tabular}

Figure 4 ROC analysis to evaluate the predictive efficiency of the nomograms for LNM and distant metastasis. ROC curves of LNM and distant metastasis in $(\mathrm{A}, \mathrm{C})$ the training cohort and $(\mathrm{B}, \mathrm{D})$ the testing cohort, respectively. LNM, lymph node metastasis; ROC, receiver operating characteristic; AUC, area under the curve; $95 \%$ CI, 95\% confidence interval.

risk of LNM than counterparts in the low-risk group (Figure S1A), which was consistent with the prediction outcome in the testing cohort (Figure S1B). Similarly, patients in the high-risk group suffered from a higher probability for distant metastasis than those in the low-risk group in both cohorts (Figure S1C,D).

\section{Clinical application of nomogram model for LNM and distant metastasis prediction}

Furthermore, survival analysis also revealed that earlystage NSCLC subjects who were predicted to develop LNM (Figure S2A,B) or distant metastasis (Figure S2C,D) 
A

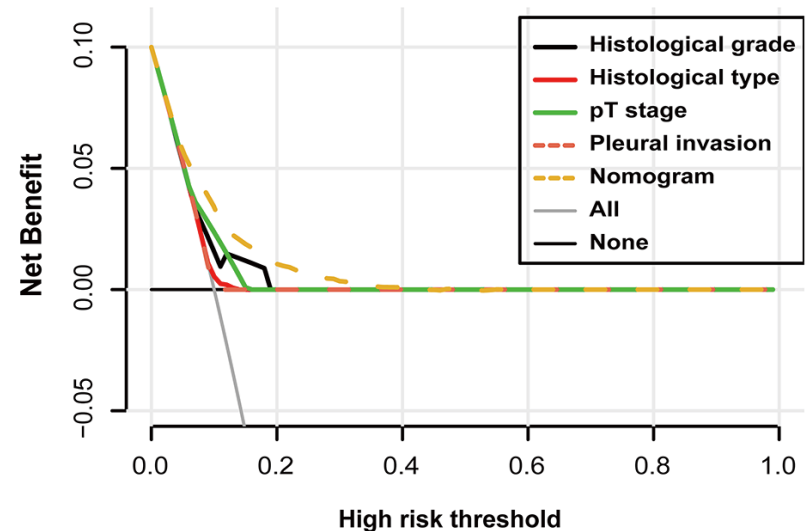

C

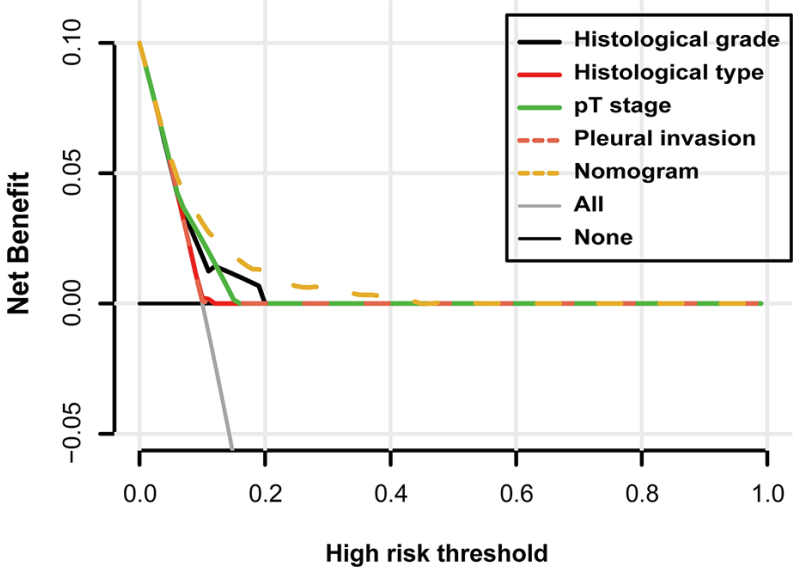



$\mathrm{D}$

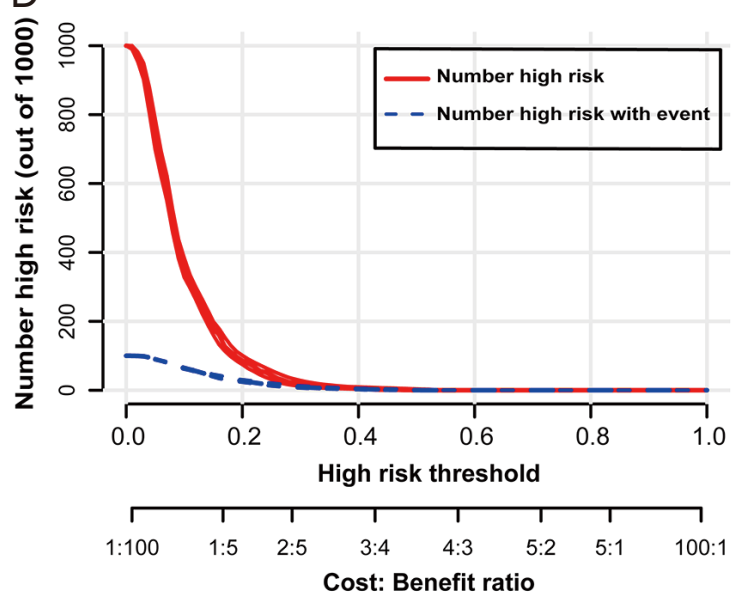

Figure 5 DCA curve and clinical impact curve of LNM nomogram in $(A, B)$ the training cohort and $(C, D)$ the testing cohort, respectively. LNM, lymph node metastasis; DCA, decision curve analysis.

were characterized with a significantly decreased OS in both cohorts $(\mathrm{P}<0.0001)$. The DCA curve demonstrated that compared with the traditional variables, the LNM nomogram in the training group displayed the optimal clinical usability and benefits, followed by histological grade, pT stage and histological type as well as pleural invasion (Figure 5). A roughly consistent trend was also revealed in the LNM nomogram in the testing group (Figure 5). Additionally, clinical impact curves illustrated when the risk threshold was within a range of 0 to 0.3 , the predicted number of early-stage NSCLC individuals with high risk appeared to be higher than the number of highrisk individuals with an actual LNM occurrence in both training and testing cohorts (Figure 5). Similarly, DCA curves showed the highest net benefit of the nomogram for predicting distant metastasis compared to the other four parameters (histological grade, LNM, pleural invasion and histological type) in both cohorts (Figure 6). As showed in Figure 6, the cost benefit ratios were admissible within the range of 0 to 0.4 .

\section{Discussion}

Lung cancer ranks first in terms of incidence and mortality worldwide in 2018. Distant metastasis is the primary reason responsible for unsatisfactory prognosis of early-stage NSCLC patients. LNM is also a considerable prognostic factor associated with tumor recurrence $(1,2,22,23)$. Thus, it is momentous to estimate the risk factors associated with LNM and distant metastasis for early-stage NSCLC patients. In our report, we initially identified the clinicopathologic parameters' difference between earlystage NSCLC patients with LNM and distant metastasis and those without both metastases. We further confirmed 
A

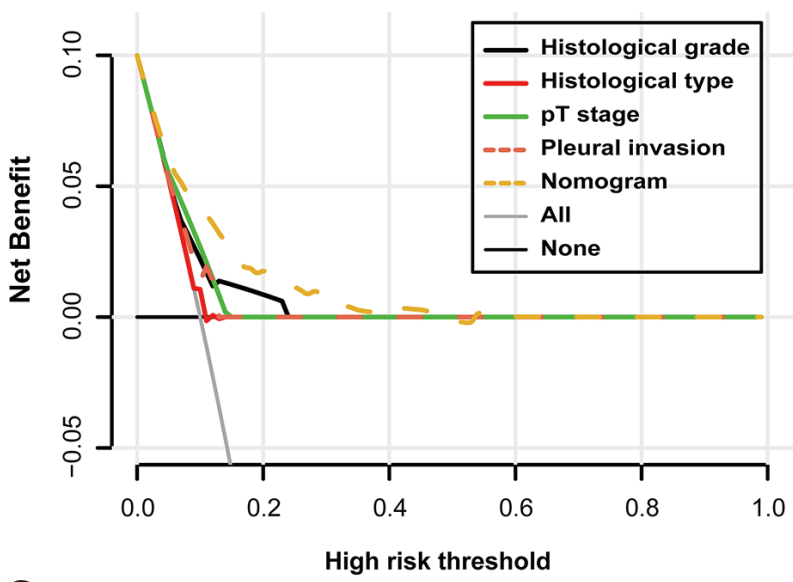

C

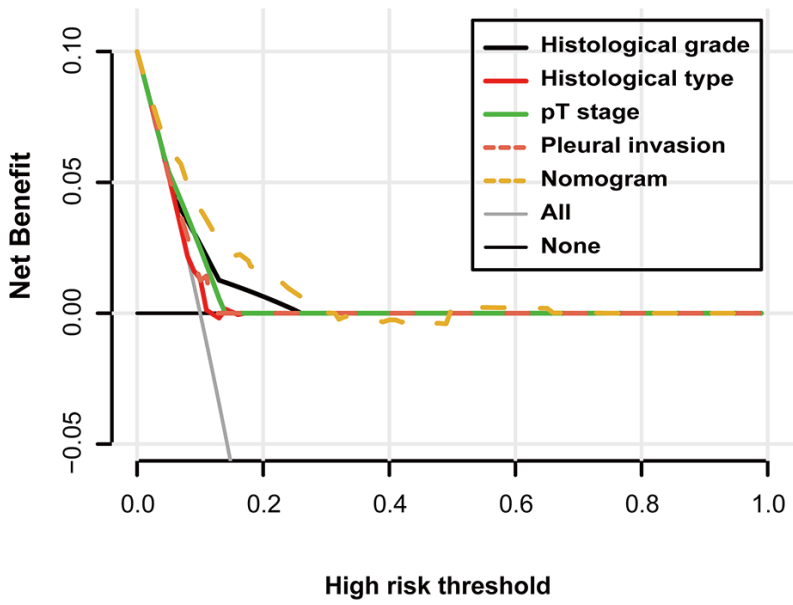

B

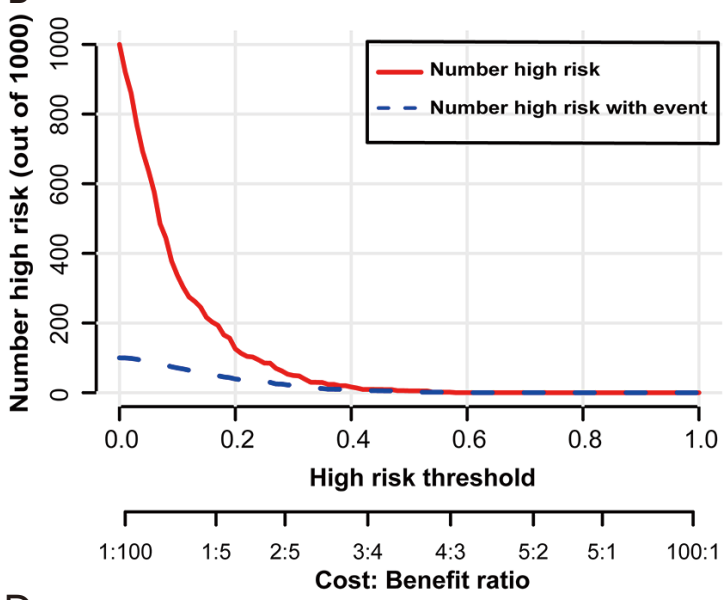

D

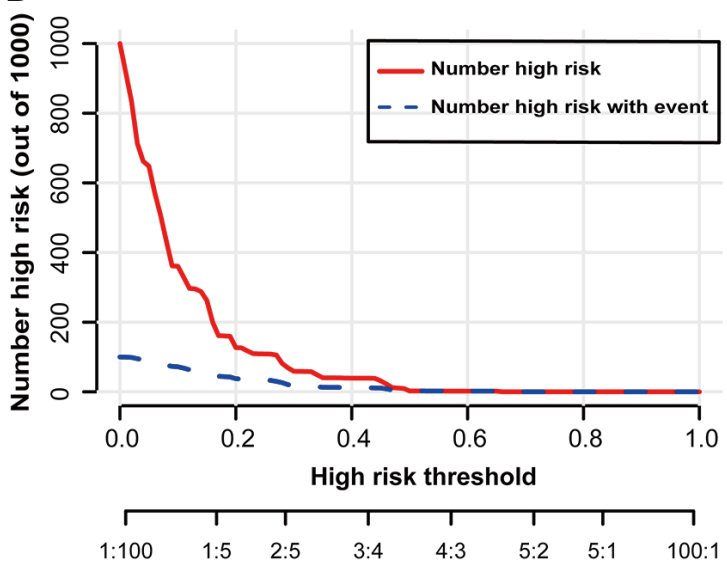

Cost: Benefit ratio

Figure 6 DCA curve and clinical impact curve of distant metastasis nomogram in (A,B) the training cohort and (C,D) the testing cohort, respectively. DCA, decision curve analysis.

certain significant clinical risk factors for LNM and distant metastasis through univariable and multivariable logistic regression analysis. Two visualized nomogram models were formulated and validated to confer prediction for the occurrence of LNM and distant metastasis in early-stage NSCLC patients through including all significant risk predictors filtrated in the multivariable logistic regression analysis. Our two nomograms revealed favorable coherence between model prediction and actual observation in the training and testing cohorts. Moreover, a satisfactory AUC value of the LNM and distant metastasis nomograms was 0.721 (95\% CI: $0.711-0.734)$ and 0.788 (95\% CI: 0.7610.816 ), respectively, in the training group, highlighting that the predictive performance of nomogram models was significantly superior to that of conventional clinical parameters, including pathological differentiation, histologic type, pT grade and the status of lymph node invasion.

Specifically, the predictive nomogram for LNM included ten clinical variables, namely surgery type, pathological differentiation, the status of distant metastasis, pT stage, histological type, tumor size, primary site of tumor and age as well as gender, which were basically consistent with the outcome in the previous clinical studies (24-27). Notably, neoplasm located in the LLL was prone to develop LNM, which was not consistent with another report's conclusion that tumor in the RLL had the most obvious tendency for LNM (27). Additionally, we also identified that several traditional and crucial clinical parameters, including pathological differentiation, 
histological type and tumor size, were all independent risk factors associated with LNM. According to our analysis, early-stage NSCLC patients with a tumor size of more than $30 \mathrm{~mm}$ and poorly differentiated or undifferentiated adenocarcinoma were more inclined to have a higher grade of lymph node invasion. Therefore, it seems that physicians take these patients more seriously than others and imaging screening is still a reliable and convenient pathway to detect metastasis (21).

The nomogram for distant metastasis incorporated seven clinical factors: the status of lymph node involvement, pathological differentiation, histological type, surgery type, the status of pleural invasion, age and sex. Similarly, Zhang et al. retrospectively analyzed 637 resected NSCLC patients and further developed a nomogram to forecast the development of distant metastasis based on three significant clinicopathologic characteristics, including histological type, pathological differentiation, degree of lymph nodes metastasis (22). Wang et al. highlighted that locally advanced NSCLC subjects with non-squamous pathological subtypes and more metastatic lymph nodes had a high risk of developing brain metastases (28). Our model also demonstrated that more aggressive LNM, NSCLC subtype adenocarcinoma and poor tumor differentiation were the primary factors responsible for distant metastases. Notably, lymph node involvement played a central role in facilitating distant metastasis in our nomogram. Indeed, a previous report also demonstrated that M1b NSCLC patients displayed higher proportions of $\mathrm{N} 1, \mathrm{~N} 2$ and $\mathrm{N} 3$ than $\mathrm{M} 1 \mathrm{a}$ patients did, and patients with aggressive $\mathrm{N}$ stage had a higher probability of developing multiorgan metastases (both $\mathrm{P}<0.001)(29,30)$. Intriguingly, our results concurred with those of several previous studies in which younger patients were characterized with an enhanced risk of lymph node invasion and distant metastasis $(31,32)$. In our cohort, male gender was an independent risk factor for developing LNM and distant metastasis in early-stage NSCLC patients. NSCLC, distinct from breast cancer, is generally acknowledged as a hormone-independent cancer. Whether gender potentially exerts a momentous effect on various metastases in hormone-independent tumors needs further investigation (21). As for histological type, our results demonstrated that adenocarcinoma exhibited a higher risk of distant metastasis than other histological subtypes. Indeed, Oliveira et al. demonstrated that adenocarcinoma was the most frequent histological subtype $(58.3 \%)$ to develop bone metastasis while squamous cell carcinoma was significantly related to the lowest risk of bone metastasis
(13.0\%) (33). A prior clinical study also found that lung adenocarcinoma occupied over $50 \%$ of all the lung cancer cases with bone metastasis (34). Another tumor registrybased research performed in Sweden revealed up to 39\% of bone metastasis occurrence in adenocarcinoma (11). Adenocarcinoma subjects also exhibited a 2.86-fold higher risk of brain metastases occurrence compared to nonadenocarcinoma NSCLC patients (22).

Our report had considerable strengths. Firstly, to our knowledge, we established the first predictive nomograms that could estimate the clinicopathological risk factors associated with LNM and distant metastasis in pT1 or pT2a NSCLC patients. Secondly, we extracted the largest number of cases with early-stage NSCLC from the SEER database and randomly classified them into a training cohort and an internal testing cohort, which made our conclusion more convincing. Thirdly, two user-friendly nomogram models performed well and harboured satisfactory accuracy and reliability. All significant risk factors in our nomograms were accessible and prevalent in clinical practice. Thus, our models were convenient for clinicians to perform the individualized risk prediction of each patient. Inevitably, our study has several limitations. Firstly, the representativeness of our sample was partial as all enrolled subjects were from the USA and clinical information concerning cases from additional countries or areas was devoid in the SEER database. Furthermore, the SEER database was devoid of certain crucial clinicopathologic factors of NSCLC, including smoking, body mass index, family history, driven mutations and genetic markers $(35,36)$, which requires further investigations. Ultimately, our predictive nomograms were merely assessed by internal verification owing to the deficiency of data support from another database of our hospital (37). Thus, the applicability of our nomograms to early-stage NSCLC patients remains to be further confirmed through multicenter prospective clinical trials with a large sample size.

\section{Conclusions}

Based on the identified clinicopathologic risk predictors in a large retrospective population-based cohort, our study presented the first novel nomogram models with favorable predictive accuracy to make an individualized distinction and prediction for LNM and distant metastasis in resected patients with pT1 and pT2a NSCLC, which is instrumental in prognostic evaluation, personalized therapy and followup management in clinical practice. 


\section{Acknowledgments}

Because our research was primarily based on the publicly available SEER database, there was no funding to support our research. We would like to thank the SEER database for its open access.

Funding: None.

\section{Footnote}

Reporting Checklist: The authors have completed the TRIPOD reporting checklist. Available at http://dx.doi. org/10.21037/apm-20-1756

Conflicts of Interest: All authors have completed the ICMJE uniform disclosure form (available at http://dx.doi. org/10.21037/apm-20-1756). The authors have no conflicts of interest to declare.

Ethical Statement: The authors are accountable for all aspects of the work in ensuring that questions related to the accuracy or integrity of any part of the work are appropriately investigated and resolved. The study was conducted in accordance with the Declaration of Helsinki (as revised in 2013). The SEER database is publicly available and our study was performed based on the guideline of this database. All patient information was anonymized and de-identified in the SEER database. Thus, our study was exempted from the ethics committee approval and patients' informed consent.

Open Access Statement: This is an Open Access article distributed in accordance with the Creative Commons Attribution-NonCommercial-NoDerivs 4.0 International License (CC BY-NC-ND 4.0), which permits the noncommercial replication and distribution of the article with the strict proviso that no changes or edits are made and the original work is properly cited (including links to both the formal publication through the relevant DOI and the license). See: https://creativecommons.org/licenses/by-nc-nd/4.0/.

\section{References}

1. Zhang $Z$, Tang $H$, Chen $P$, et al. Demystifying the manipulation of host immunity, metabolism, and extraintestinal tumors by the gut microbiome. Signal Transduct Target Ther 2019;4:41.

2. Bray F, Ferlay J, Soerjomataram I, et al. Global cancer statistics 2018: GLOBOCAN estimates of incidence and mortality worldwide for 36 cancers in 185 countries. CA Cancer J Clin 2018;68:394-424.

3. Rotow J, Bivona TG. Understanding and targeting resistance mechanisms in NSCLC. Nat Rev Cancer 2017;17:637-58.

4. Thomas A, Liu SV, Subramaniam DS, et al. Refining the treatment of NSCLC according to histological and molecular subtypes. Nat Rev Clin Oncol 2015;12:511-26.

5. Liu WJ, Du Y, Wen R, et al. Drug resistance to targeted therapeutic strategies in non-small cell lung cancer. Pharmacol Ther 2020;206:107438.

6. Hirsch FR, Scagliotti GV, Mulshine JL, et al. Lung cancer: current therapies and new targeted treatments. Lancet 2017;389:299-311.

7. Zhang $\mathrm{Z}$, Chen $\mathrm{P}$, Xie $\mathrm{H}$, et al. Using circulating tumor DNA as a novel biomarker to screen and diagnose hepatocellular carcinoma: A systematic review and metaanalysis. Cancer Med 2020;9:1349-64.

8. Tan WL, Chua KLM, Lin CC, et al. Asian Thoracic Oncology Research Group expert consensus statement on optimal management of stage III NSCLC. J Thorac Oncol 2020;15:324-43.

9. Diederich S. Lung cancer staging update: the revised TNM classification. Cancer Imaging 2010;10 Spec no A:S134-5.

10. Goldstraw P. The 7th Edition of TNM in Lung Cancer: what now? J Thorac Oncol 2009;4:671-3.

11. Riihimaki M, Hemminki A, Fallah M, et al. Metastatic sites and survival in lung cancer. Lung Cancer 2014;86:78-84.

12. Li J, Zhu H, Sun L, et al. Prognostic value of site-specific metastases in lung cancer: A population based study. $\mathrm{J}$ Cancer 2019;10:3079-86.

13. Ruffini E, Asioli S, Filosso PL, et al. Significance of the presence of microscopic vascular invasion after complete resection of Stage I-II pT1-T2N0 non-small cell lung cancer and its relation with T-Size categories: did the 2009 7th edition of the TNM staging system miss something? J Thorac Oncol 2011;6:319-26.

14. Goldstraw P, Chansky K, Crowley J, et al. The IASLC Lung Cancer Staging Project: Proposals for Revision of the TNM Stage Groupings in the Forthcoming (Eighth) Edition of the TNM Classification for Lung Cancer. J Thorac Oncol 2016;11:39-51.

15. Ashour Badawy A, Khedr G, Omar A, et al. Site of Metastases as Prognostic Factors in Unselected Population of Stage IV Non-Small Cell Lung Cancer. Asian Pac J 
Cancer Prev 2018;19:1907-10.

16. Zhu WY, Fang KX, He JY, et al. A Prediction Rule for Overall Survival in Non-Small-Cell Lung Cancer Patients with a Pathological Tumor Size Less Than $30 \mathrm{~mm}$. Dis Markers 2019;2019:8435893.

17. Kluth LA, Black PC, Bochner BH, et al. Prognostic and Prediction Tools in Bladder Cancer: A Comprehensive Review of the Literature. Eur Urol 2015;68:238-53.

18. Balachandran VP, Gonen M, Smith JJ, et al. Nomograms in oncology: more than meets the eye. Lancet Oncol 2015;16:e173-80.

19. Furqan M, Tien YY, Schroeder MC, et al. Lobar versus sub-lobar surgery for pulmonary typical carcinoid, a population-based analysis. J Thorac Dis 2018;10:5850-9.

20. Zhang Z, Xie H, Chen P, et al. Development and Identification of a Nomogram Prognostic Model for Patients with Primary Clear Cell Carcinoma of the Liver. Med Sci Monit 2020;26:e919789.

21. Mok TSK, Wu YL, Kudaba I, et al. Pembrolizumab versus chemotherapy for previously untreated, PD-L1expressing, locally advanced or metastatic non-small-cell lung cancer (KEYNOTE-042): a randomised, open-label, controlled, phase 3 trial. Lancet 2019;393:1819-30.

22. Zhang F, Zheng $W$, Ying L, et al. A Nomogram to Predict Brain Metastases of Resected Non-Small Cell Lung Cancer Patients. Ann Surg Oncol 2016;23:3033-9.

23. Yang X, Pan X, Liu H, et al. A new approach to predict lymph node metastasis in solid lung adenocarcinoma: a radiomics nomogram. J Thorac Dis 2018;10:S807-S819.

24. Cerfolio RJ, Bryant AS, Eloubeidi MA. Routine mediastinoscopy and esophageal ultrasound fine-needle aspiration in patients with non-small cell lung cancer who are clinically N2 negative: a prospective study. Chest 2006;130:1791-5.

25. Gomez-Caro A, Garcia S, Reguart N, et al. Incidence of occult mediastinal node involvement in cN0 non-smallcell lung cancer patients after negative uptake of positron emission tomography/computer tomography scan. Eur J Cardiothorac Surg 2010;37:1168-74.

26. Shafazand S, Gould MK. A clinical prediction rule to estimate the probability of mediastinal metastasis in patients with non-small cell lung cancer. J Thorac Oncol 2006;1:953-9.

27. Jiang L, Jiang S, Lin Y, et al. Nomogram to Predict Occult N2 Lymph Nodes Metastases in Patients With Squamous
Nonsmall Cell Lung Cancer. Medicine (Baltimore) 2015;94:e2054.

28. Wang SY, Ye X, Ou W, et al. Risk of cerebral metastases for postoperative locally advanced non-small-cell lung cancer. Lung Cancer 2009;64:238-43.

29. Yang J, Peng A, Wang B, et al. The prognostic impact of lymph node metastasis in patients with non-small cell lung cancer and distant organ metastasis. Clin Exp Metastasis 2019;36:457-66.

30. Dai C, Ren Y, Xie D, et al. Does Lymph Node Metastasis Have a Negative Prognostic Impact in Patients with NSCLC and M1a Disease? J Thorac Oncol 2016;11:1745-54.

31. Chen T, Zhou F, Jiang W, et al. Age at diagnosis is a heterogeneous factor for non-small cell lung cancer patients. J Thorac Dis 2019;11:2251-66.

32. Xia W, Wang A, Jin $M$, et al. Young age increases risk for lymph node positivity but decreases risk for non-small cell lung cancer death. Cancer Manag Res 2018;10:41-8.

33. Oliveira MB, Mello FC, Paschoal ME. The relationship between lung cancer histology and the clinicopathological characteristics of bone metastases. Lung Cancer 2016;96:19-24.

34. Sugiura H, Yamada K, Sugiura T, et al. Predictors of survival in patients with bone metastasis of lung cancer. Clin Orthop Relat Res 2008;466:729-36.

35. Fillon $M$. Tobacco control initiatives cut the number of lung cancer deaths in California by 28. CA Cancer J Clin 2019;69:83-5.

36. Karuppasamy R, Veerappapillai S, Maiti S, et al. Current progress and future perspectives of polypharmacology: From the view of non-small cell lung cancer. Semin Cancer Biol 2021;68:84-91.

37. Zheng XQ, Huang JF, Lin JL, et al. Incidence, prognostic factors, and a nomogram of lung cancer with bone metastasis at initial diagnosis: a population-based study. Transl Lung Cancer Res 2019;8:367-79.

Cite this article as: Tian Y, He Y, Li X, Liu X. Novel nomograms to predict lymph node metastasis and distant metastasis in resected patients with early-stage non-small cell lung cancer. Ann Palliat Med 2021;10(3):2548-2566. doi: 10.21037/ apm-20-1756 


\section{Supplementary}

Table S1 Clinical features of the included early-stage NSCLC patients



Table S1 (continued) 
Table S1 (continued)

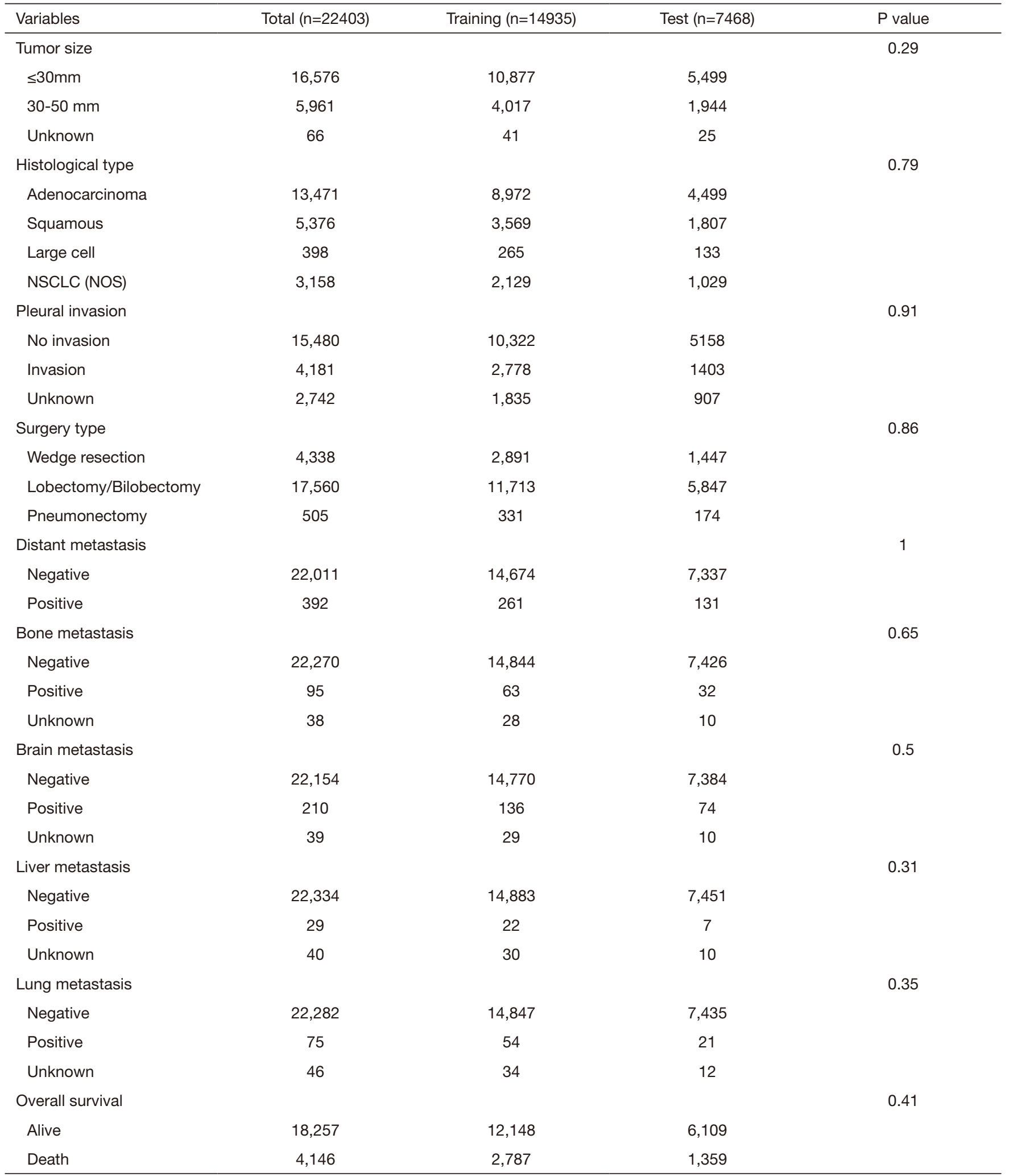

*, Nx means that there is lymph node metastasis, but unknown for which stage. SD, standard deviation; RUL, right upper lobe; RML, right middle lobe; RLL, right lower lobe; LUL, left upper lobe; LLL, left lower lobe; pT, pathologic T; NSCLC (NOS), Non-small cell lung cancer, Not otherwise specified. 
Table S2 Point distributions and predictive scores for every parameter in both nomograms

\begin{tabular}{|c|c|c|}
\hline \multirow[b]{2}{*}{ Variables } & \multicolumn{2}{|c|}{ Nomogram score } \\
\hline & LNM & $\begin{array}{c}\text { Distant } \\
\text { metastasis }\end{array}$ \\
\hline \multicolumn{3}{|l|}{ Age } \\
\hline$\leq 65$ & 12 & 23 \\
\hline$>65$ & 0 & 0 \\
\hline \multicolumn{3}{|l|}{ Gender } \\
\hline Female & 0 & 0 \\
\hline Male & 5 & 13 \\
\hline \multicolumn{3}{|l|}{ Primary site } \\
\hline RUL & 0 & - \\
\hline RML & 13 & - \\
\hline RLL & 13 & - \\
\hline LUL & 14 & - \\
\hline LLL & 15 & - \\
\hline \multicolumn{3}{|c|}{ Pathological differentiation } \\
\hline Well; I & 0 & 0 \\
\hline Moderately; II & 51 & 40 \\
\hline Poorly; III & 64 & 59 \\
\hline Undifferentiated; IV & 67 & 70 \\
\hline \multicolumn{3}{|l|}{ pT stage } \\
\hline $\mathrm{T} 1$ & 0 & - \\
\hline $\mathrm{T} 2 \mathrm{a}$ & 25 & - \\
\hline \multicolumn{3}{|l|}{ Lymph node status } \\
\hline No & - & 0 \\
\hline N1 & - & 25 \\
\hline N2 & - & 42 \\
\hline N3 & - & 100 \\
\hline
\end{tabular}

Table S2 (continued)
Table S2 (continued)

\begin{tabular}{|c|c|c|}
\hline \multirow[b]{2}{*}{ Variables } & \multicolumn{2}{|c|}{ Nomogram score } \\
\hline & LNM & $\begin{array}{c}\text { Distant } \\
\text { metastasis }\end{array}$ \\
\hline \multicolumn{3}{|l|}{ Tumor size } \\
\hline$\leq 30 \mathrm{~mm}$ & 0 & - \\
\hline $30-50 \mathrm{~mm}$ & 16 & - \\
\hline \multicolumn{3}{|l|}{ Histological type } \\
\hline Adenocarcinoma & 23 & 30 \\
\hline Squamous & 0 & 0 \\
\hline Large cell & 9 & 42 \\
\hline NSCLC (NOS) & 14 & 26 \\
\hline \multicolumn{3}{|l|}{ Pleural invasion } \\
\hline No invasion & - & 0 \\
\hline Invasion & - & 22 \\
\hline \multicolumn{3}{|l|}{ Surgery type } \\
\hline Wedge resection & 0 & 39 \\
\hline Lobectomy/bilobectomy & 28 & 1 \\
\hline Pneumonectomy & 100 & 0 \\
\hline \multicolumn{3}{|l|}{ Distant metastasis } \\
\hline Negative & 0 & - \\
\hline Positive & 52 & - \\
\hline
\end{tabular}


A

Lymph node metastasis-nomogram

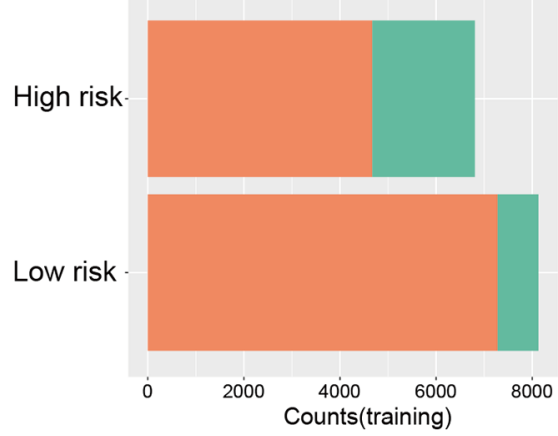

C

Distant metastasis-nomogram

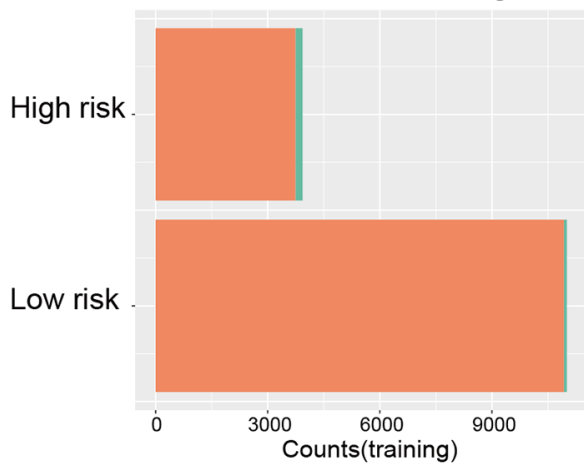

B

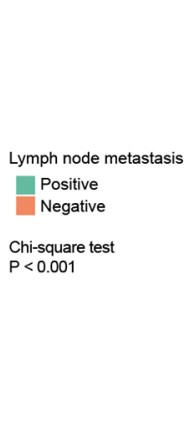

$\mathrm{D}$

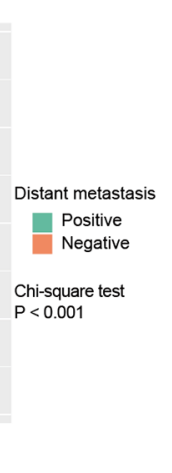

Lymph node metastasis-nomogram
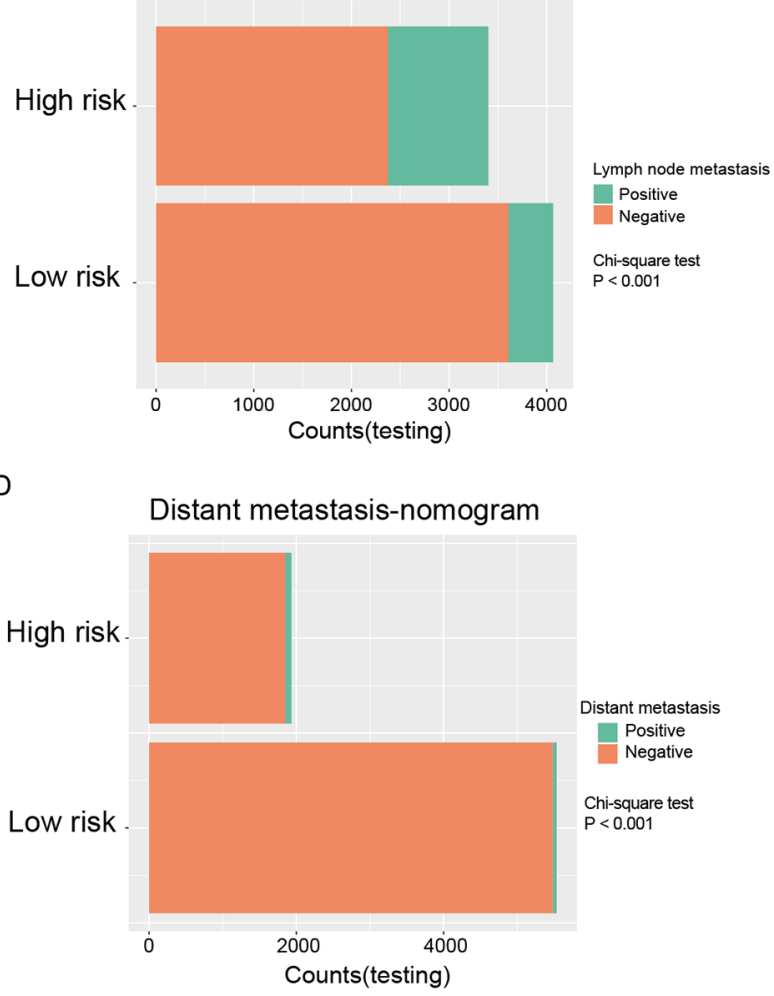

Figure S1 Discriminatory efficiency of the predictive nomogram models for LNM and distant metastasis. Risk stratification for the nomogram models was performed through the maximum Youden index, and their performance in differentiating LNM and distant metastasis in $(\mathrm{A}, \mathrm{C})$ the training cohort and $(\mathrm{B}, \mathrm{D})$ the testing cohort were plotted. 

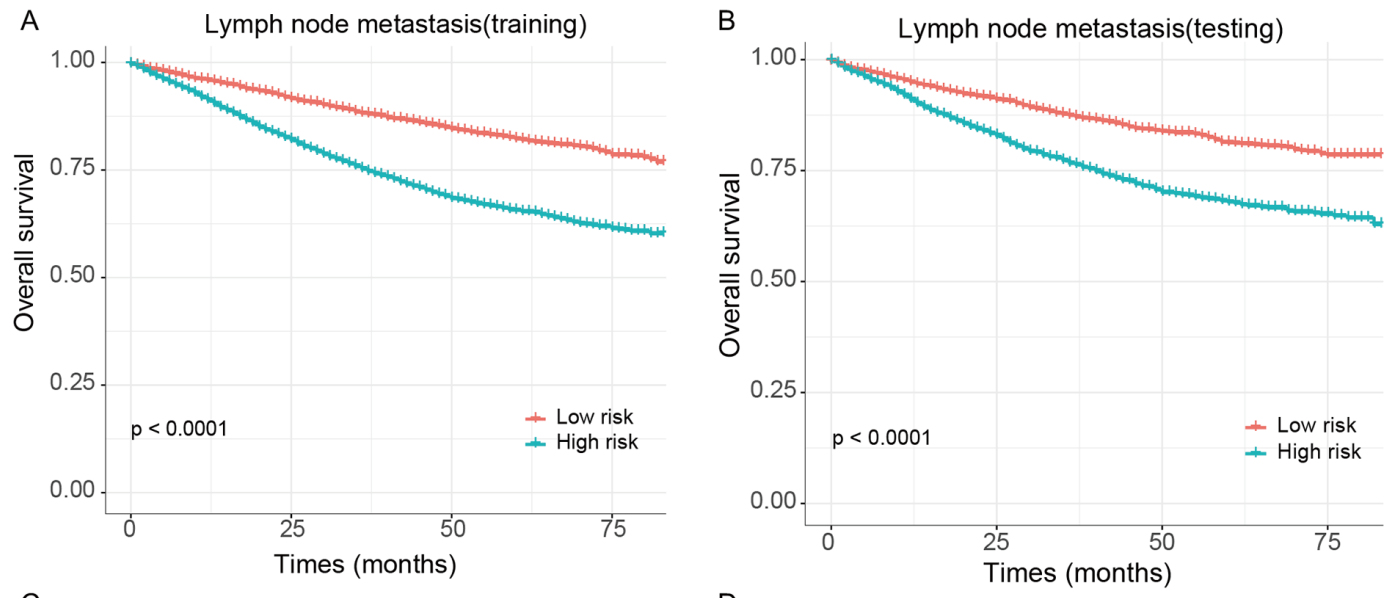

C

D
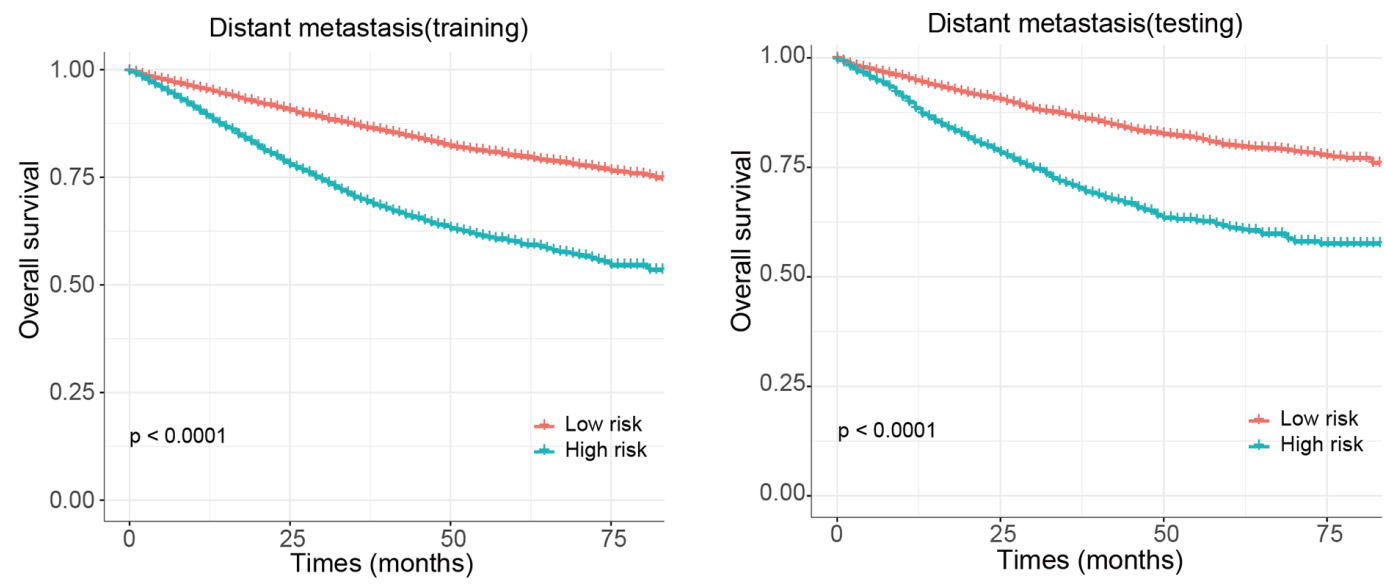

Figure S2 Survival analysis of patients with LNM and distant metastasis in $(A, C)$ the training cohort and (B,D) the testing cohort, respectively. OS, overall survival. 